\title{
Treatment of Idiopathic Intracranial Hypertension by Single Tuohy Needle Lumbar Puncture
}

\author{
AHMED E. ABOKRESHA, M.D. \\ The Department of Neurosurgery, Faculty of Medicine, Assiut University, Assiut, Egypt
}

\begin{abstract}
Background: Idiopathic Intracranial Hypertension (IIH) is seen typically in obese women in their child-bearing age, management of patients with IIH can be problematic.

Aim of Study: The purpose of this study is to assess single spinal tapping using wide pore Tuohy needle as a modality for treatment of IIH.

Objective: The purpose of this study is to assess single Tuohy needle spinal tapping as a modality for treatment of IIH.

Patients and Methods: This was a prospective case series of twenty women with IIH who underwent full neurological and ophthalmological evaluation including fluoruscopic fundus examination and neuroimaging studies. They were followedup and treated using only single Tuohy needle spinal tapping.

Results: Single spinal tapping can control IIH safely with a success rate of $100 \%$.

Conclusion: Single spinal tapping using Tuohy needle is recommended as a treatment modality in women with IIH.
\end{abstract}

Key Words: Idiopathic intracranial hypertension - Lumbar puncture - Tuohy needle.

\section{Introduction}

IDIOPATHIC Intracranial Hypertension (IIH) is a syndrome in which Intracranial Pressure (ICP) is elevated with normal Cerebrospinal Fluid (CSF) composition and no evidence of hydrocephalus or mass lesion. It is seen typically in obese women of child-bearing age with annual incidence of 19.3 per 100,000 [1].

The most common symptoms of IIH are headache, occurring in $90 \%$ of cases and visual disturbance including transient visual obscurations and diplopia [2,3]. The typical sign is papilledema [4]

Correspondence to: Dr. Ahmed E. Abokresha, E-Mail: Ahabokreshaa@gmail.com
Diagnosis of IIH is made by exclusion using the modified Dandy criteria (Table 1) which require an increased ICP $>25 \mathrm{CmH} 2 \mathrm{O}$ and ruling out other pathologies of CSF examination and radiological imaging [4].

The pathophysiological mechanisms causing increased ICP for patients with IIH are not well understood. Possible causes include excess production of CSF, decreased CSF absorption, and increased cerebral venous sinus pressure [5]. Since IIH particularly affects obese women, it was suggested that hormones may play a role in the pathogenesis of the disease [2].

The first-line of treatment usually emphasizes weight loss by diet and the use of carbonic anhydrase inhibitors [6].

The purpose of this study is to assess single spinal tapping using wide pore Tuohy needle as a modality for treatment of IIH.

Table (1): Modified Dandy criteria [4]

- Signs and/or symptoms of increased ICP.

- Documented elevated intracranial pressure (>25CmH 2O).

- Normal CSF composition.

- No evidence of hydrocephalus, mass, structural, or vascular lesion on imaging.

- No locallizing neurological signs expect a unilateral/bilateral VI nerve paresis.

CSF: Cerebrospinal Fluid; Quoted from Friedman and Jocobson [4]

\section{Patients and Methods}

This was a prospective case series of twenty women (25-50 years old) diagnosed with IIH according to the modified Dandy criteria [4] managed at Assiut University Hospitals undertaken by a single neurosurgeon between 2013 and 2018. The patients underwent full neurological and ophthalmological evaluation including visual acuity and 
fundus examination. Neuroimaging studies including brain MRI and MRV were done to exclude mass lesions, hydrocephalus or other pathologies.

All patients were treated with single Lumbar Puncture (LP) using a wide pore Tuohy needle (16 Guage). LP was performed under local anesthesia using $20-\mathrm{G}$ needle that was inserted under complete aseptic precautions in L4-L5 vertebral space. The opening pressure was measured followed by continuous CSF drainage till 30-40CC is obtained.

\section{Results}

Follow-up of the patients clinically according to headache and blurring of vision and ophthalmologically according to papillaedema was the guide which show that headache and blurring of vision improved immediately and papillaedema improved 2 weeks after LP. Of the 20 cases only 3 complaint of low tension headache which improved by putting the patient in the supine position with the head low down for 3 days and steriods.

\section{Discussion}

The exact etiology of IIH is unknown. Although it is often considered to be idiopathic, detailed investigation has revealed a high incidence of venous drainage abnormalities in IIH patients [7] At least two primary mechanisms for the increased CSF pressure in IIH have been proposed and supported by experimental data, increased CSF production causing vasogenic extracellular brain edema and a low CSF outflow at the arachnoid villi [8].

It was found in this study that 17 patients $(85 \%)$ were overweight which supports the notion that obesity could contribute to the pathogenesis of IIH. The postulated mechanism is that central obesity raises the intra-abdominal pressure, which elevates pleural pressure and cardiac filling pressures, which hinders venous return from the brain and leads to elevated intracranial venous pressure and elevated intracranial pressure [9].

In IIH patients, weight loss and diet are considered integral parts of IIH treatment.

Acetazolamide is considered the first-line medical treatment for $\mathrm{IIH}$.

Use [10]. Other medical treatments indicated for IIH include furosemide and steroids. Furosemide decreases intracranial pressure by diuresis and by reduction of the transport of sodium ions in the brain.
LP and spinal tapping is an effective tool in management of patients with IIH as it directly reduces intracranial pressure immediately [11]. It allows CSF drainage and reduces CSF pressure.

In this study, all patients underwent single LP and spinal tapping using Tuohy needle as a modality to relieve CSF pressure; it was found that all patients showed clinical improvement as regard to headache and blurring of vision and ophthalmological examination as regard to papilledema.

Three patients of these cases complaint of low tension headache relieved by by putting the patient in the supine position with the head low down for 3 days and steriods.

Mohamed I. Hegazy et al., [12] found that 57\% of cases with IIH needed single LP and showed clinical improvement on ophthalmological examination as the papilledema improved and optic disc borders became more distinct.

LP and spinal tapping is considered to be the treatment of choice in patients with IIH.

\section{Conclusion:}

Single LP and spinal fluid tapping using Tuohy needle can control IIH safely with a success rate of $85 \%$.

\section{References}

1- BADVE M., McCONNELL M., SHAH T., ONDECKOLIGDA K., POUTOUS G., VALLEJO M., et al.: Idiopathic intracranial hypertension in pregnancy treated with serial lumbar punctures. Int. J. Clin. Med., 2: 9-12, 2011.

2- KESLER A. and KUPFERMINC M.: Idiopathic intracranial hypertension and pregnancy. Clin. Obstet. Gynecol., 56: 389-96, 2013.

3- HUNA-BARON R. and KUPERSMITH M.J.: Idiopathic intracranial hypertension in pregnancy. J. Neurol., 249: 1078-81, 2002.

4- FRIEDMAN D.I. and JACOBSON D.M.: Diagnostic criteria for idiopathic intracranial hypertension. Neurology, 59: 1492-5, 2002.

5- DURCAN F.J., CORBETT J.J. and WALL M.: The incidence of pseudotumor cerebri: Population studies in Iowa and Louisiana. Arch. Neurol., 45: 875-7, 1988.

6- KUPERSMITH M.J., GAMELL L., TURBIN R., PECK V., SPIEGEL P. and WALL M.: Effects of weight loss on the course of idiopathic intracranial hypertension in women. Neurology, 50: 1094-8, 1998.

7- BRAZIS P.W.: Pseudotumor cerebri. Curr. Neurol. Neurosci. Rep., 4: 111-6, 2004.

8- RADHAKRISHNAN K., AHLSKOG J.E., GARRITY J.A. and KURLAND L.T.: Idiopathic intracranial hypertension. Mayo Clin. Proc., 69: 169-80, 1994. 
9- BAGGA R., JAIN V., DAS C.P., GUPTA K.R., GOPALAN S. and MALHOTRA S.: Choice of therapy and mode of delivery in idiopathic intracranial hypertension during pregnancy. Medscape Gen. Med., 7: 42-6, 2005.

10- DIGRE K.B., VARNER M.W. and CORBETT J.J.: Pseudotumor cerebri and pregnancy. Neurology, 34: 721-9, 1988.

11-ABOULEISH E., ALI V. and TANG R.A.: Benign intrac- ranial hypertension and anesthesia for cesarean section. Anesthesiology, 63: 9705-7, 1985.

12- MOHAMED I. HEGAZY, EHAB MOHAMED EISSAA, LAMIAA IBRAHIM DAKERD and MONA MOHAMED SHABANB: Lumbar puncture as a single modality for treatment of idiopathic intracranial hypertension during pregnancy. The Egyptian Journal of Neurology, Psychiatry and Neurosurgery, 53 (1): 33-6, 2016.

\section{علاج إرتفاع ضغط الميخ مجهول السبب

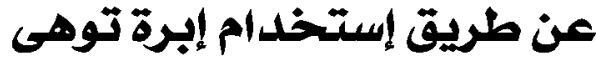

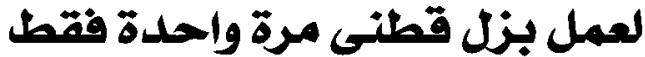

عمل بزل قطنى مرة واحدة فقط وإنسكاب السائل النخاعى بإستخدام إبرة توهى

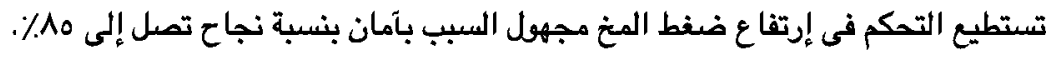

